\title{
Flux Separation in Photospheric Magnetoconvection
}

\author{
N. O. Weiss \& M. R. E. Proctor \\ Dept. of Applied Mathematics and Theoretcal Physics, University of \\ Cambridge, Cambridge CB3 $9 E W, U K$
}

\begin{abstract}
Numerical experiments on three-dimensional magnetoconvection in a stratified compressible layer reveal a range of different patterns, depending on the strength of the imposed magnetic field. As the field is decreased there is a transition from small-scale plumes, in the magnetically dominated regime, to large-scale vigorous plumes when the field is dominated by the motion. In the intermediate regime magnetic flux separates from the motion, so that there are almost field-free regions, with clusters of vigorous plumes, surrounded by regions where the Lorentz force is strong enough to control the dynamics. There is a range of field strengths where either small-scale plumes or flux-separated solutions can persist, depending on initial conditions for the computation. These results can be related to magnetic features at the surface of the Sun.
\end{abstract}

\section{The Model Calculation}

Computations are now yielding results that can be compared with the high resolution observations of granulation and intergranular magnetic fields obtained at La Palma, and related to the slender loops revealed by TRACE. We summarize here the results of a systematic investigation of three-dimensional magnetoconvection in a fully compressible, perfect gas, governed by nonlinear partial differential equations with diffusion explicitly included (Weiss et al. 1996; Rucklidge et al. 2000). We consider a plane horizontal layer with an imposed mean vertical field $\mathbf{B}_{0}$, measured by the Chandrasekhar number $Q \propto B_{0}^{2}$. The static (non-convecting) solution is a stratified polytrope, with a density that increases by a factor of 11 across the layer. The thermal and electrical conductivities and dynamic viscosity are constant; the diffusivity ratio $\zeta$ increases with depth $(0.2 \leq \zeta \leq 2.2)$, to mimic effects of ionization in the Sun; the Prandtl number $\sigma=1$.

We solve the equations in a square box with idealized boundary conditions: all quantities are periodic in horizontal directions; the field is kept vertical at the top and bottom, with impermeable stress-free boundaries; the temperature at the base is fixed, with a 'radiative' thermal boundary condition (Blanchflower et al. 1998) at the top. The superadiabatic gradient is chosen so that the Rayleigh number $R=10^{5}$, and runs are carried out for different values of $B_{0}$, with $3000 \geq Q \geq 200$. A large aspect ratio (normalized box width) $\lambda$ is essential for such calculations; nearly all runs have $\lambda=8$ and require up to $256 \times 256 \times 100$ 

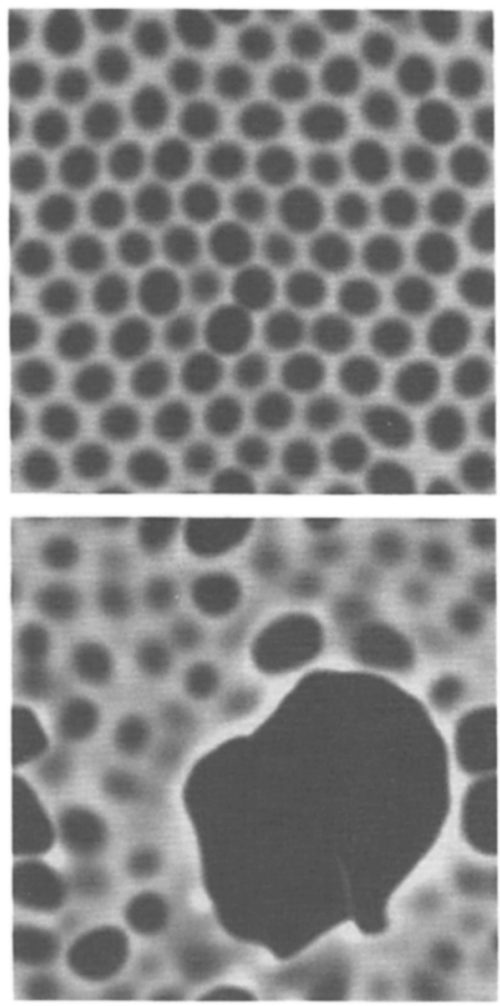

Figure 1. Hysteresis with different patterns of convection for the same imposed field. Variation of $|\mathbf{B}|^{2}$ across the top of the layer for $Q=$ $1600, \lambda=8$. Dark (light) regions denote weak (strong) fields. In the upper panel small-scale plumes expel magnetic flux to form a magnetic network. The lower panel shows an example of flux separation.

mesh points. Numerical results are obtained using a mixed finite differencepseudospectral code on the Hitachi SR-2201 supercomputer at the University of Cambridge High Performance Computing Facility.

\section{Results}

In the presence of very strong fields $(Q \gg 4000)$ convection is completely suppressed. For $4000 \geq Q \geq 2200$ there is a magnetically dominated regime where strong fields allow weak but steady convection with a stable pattern of small rising plumes on a hexagonal lattice. This is followed by an intermediate regime $(2000 \geq Q \geq 1600)$ : small plumes are still stable but there is hysteresis, as shown in figure 1 . With suitably chosen initial conditions flux separation occurs and there is another stable solution with a clump of large time-dependent plumes from which the magnetic field is excluded, while small-scale plumes persist in the surrounding region where the field strength is enhanced. As $Q$ is increased, 
the clump shrinks in size and disappears entirely for $Q \geq 2200$. For moderate field strengths $(1400 \geq Q \geq 500)$, only flux-separated solutions are found. As $Q$ is decreased, the pattern is increasingly dominated by clumps of broad convective plumes, which expand as they rise through the stratified layer, and the strong-field regions are compressed into a network that surrounds these clumps (Tao et al. 1998).

Finally, for weak fields $(Q \leq 200)$, the pattern is dominated by vigorous convection, which confines magnetic flux to a network with locally strong magnetic fields. The flux moves rapidly, like a 'magnetic fluid', within this network. Intense fields appear at nodes but they do not persist and there are no isolated flux tubes. In the weak field limit turbulent convection can act as a small-scale dynamo, maintaining a disordered magnetic field with no net vertical flux. Cattaneo (1999) has demonstrated this process, in the Boussinesq approximation.

\section{Solar Magnetic Fields}

These computational results help to clarify magnetic behaviour at the surface of the Sun. In sunspots $\left(B_{0} \approx 3000 \mathrm{G}\right)$, energy transport below the photosphere is predominantly by convection but within the umbrae of large spots there are dark nuclei with no signs of convective activity. Deeper down there must be slender plumes (as in our magnetically dominated regime) which are masked by radiative damping at the surface. Elsewhere in sunspot umbrae there are isolated bright features known as umbral dots. These apparently correspond to vigorous plumes, powerful enough to penetrate the radiative blanket, that appear through flux separation in the intermediate regime. In plage regions $\left(B_{0} \approx 150 \mathrm{G}\right)$, magnetic fields form a perforated network, which only allows abnormal convection, with smaller plumes than in the ambient field-free photosphere. Plages are prevented by the surrounding granules from diffusing outwards. They correspond to flux separation in a moderate field regine. In the quiet sun any magnetic flux is confined to intergranular lanes, as in the weak field regime; ephemeral concentrations, with fields that are locally intense, show up as bright points. The supergranular network is maintained by ephemeral active regions, which are generated by small-scale dynamo action at some depth below the solar surface.

Acknowledgments. This research is supported by the UK Particle Physics and Astronomy Research Council. We are grateful for advice and assistance from S.M. Blanchflower, F. Cattaneo, P.C. Matthews, G. McMullan, A.M. Rucklidge and L.Tao.

\section{References}

Blanchflower, S.M., Rucklidge, A.M. \& Weiss, N.O. 1998 MNRAS 301, 593

Cattaneo, F. 1999, ApJ 515, L39

Rucklidge, A.M., Weiss, N.O., Brownjohn, D.P., Matthews, P.C. \& Proctor, M.R.E. 2000, J. Fluid Mech. 419, 283

Tao, L.L., Weiss, N.O., Brownjohn, D.P. \& Proctor, M.R.E. 1998, ApJ 496, L39

Weiss, N.O., Brownjohn, D.P., Matthews, P.C. \& Proctor, M.R.E. 1996, MNRAS 283, 1153 